\title{
ANALYSIS OF ISO 6983 NC DATA BASED ON ISO 14649 CNC DATA MODEL
}

\author{
Hiroshi Yamada, Fumiki Tanaka, Masahiko Onosato \\ Graduate School of Information Science and Technology, Hokkaido University, Japan; \\ Email: yamada@dse.ssi.ist.hokudai.ac.jp.
}

\begin{abstract}
Retaining technical information is an important element for performing high quality machining. However, actual NC data (ISO 6983) contain implicit technical information which reflect the skilled worker's knowledge. In this research, for obtaining technical information, $\mathrm{NC}$ data are analyzed based on a next-generation CNC control language (ISO 14649) which contains technical information explicitly. In this paper, we demonstrate how ISO $6983 \mathrm{NC}$ data generated by CAM software are applied to the test implemented system.
\end{abstract}

Key words: Data Analysis, Technical Information, ISO 14649, NC data

\section{INTRODUCTION}

Process planning is a time-consuming and highly skilled job, and $\mathrm{t}$ Retaining technical information is an important element for performing high quality machining. Manual collection of proprietary technical information is the most popular method of generating a database. Few research works have dealt with the generation of technical information databases automatically from NC data ${ }^{1,2}$. However, actual NC data (ISO 6983) ${ }^{3}$ contain implicit technical information which reflects the skilled worker's knowledge. In this research, as shown in Figure 1, in order to obtain technical information, NC data are analyzed based on a next-generation CNC control language (ISO $14649)^{4}$ which contains technical information explicitly. The new ISO 14649 data model includes rich information such as machining features, machining operation, and machining conditions enabling feature-based programming. In this research, the machining features and process information are extracted from an NC program based on form-shaping function ${ }^{5}$.

Please use the following format when citing this chapter:

Yamada, Hiroshi, Tanaka, Fumiki, Onosato, Masahiko, 2006, in International Federation for Information Processing (IFIP), Volume 207, Knowledge Enterprise: Intelligent Strategies In Product Design, Manufacturing, and Management, eds. K. Wang, Kovacs G., Wozny M., Fang M., (Boston: Springer), pp. 109-114. 


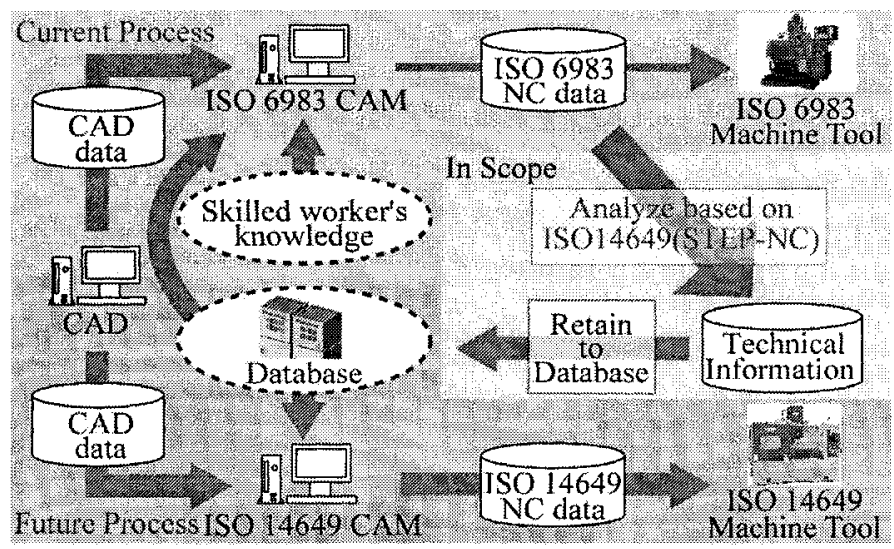

Figure 1. Objective of this research

\section{ANALYSIS OF NC DATA BASED ON ISO 14649}

\subsection{Transformation procedure from ISO 6983 NC Data to ISO 14649 workingstep}

The analysis procedure consists of five sub-procedures (activities) as shown in Figure 2. ISO $6983 \mathrm{NC}$ data, cutting tool information and the workpiece blank model are used for the analysis.

The first sub-activity is the activity splitting ISO $6983 \mathrm{NC}$ data into tool path elements for a cutting tool based on a tool change instruction, and classifying each tool path element as a bounded tool path. The classification of the tool path is based on the degree of freedom of the tool path element. The second sub-activity is generating the machining feature from the bounded tool path, the workpiece blank model, and the tool information. The details of this activity are described in 2.2. The third sub-activity is generating the machining strategy and approach-retract strategy from the bounded tool path, the approach-retract path and the workpiece blank model. These are generated based on their classification and the mapping table between the bounded tool path and the machining strategy. The fourth subactivity is generating the ISO 14649 workingstep from the tool information and the generated ISO 14649 machining feature, machining strategy, and approach-retract strategy. The fifth sub-activity is associating with the 
generated ISO 14649 workingsteps. The details of this activity are described in 2.3. Figure 2 also shows the analysis example of slot machining.

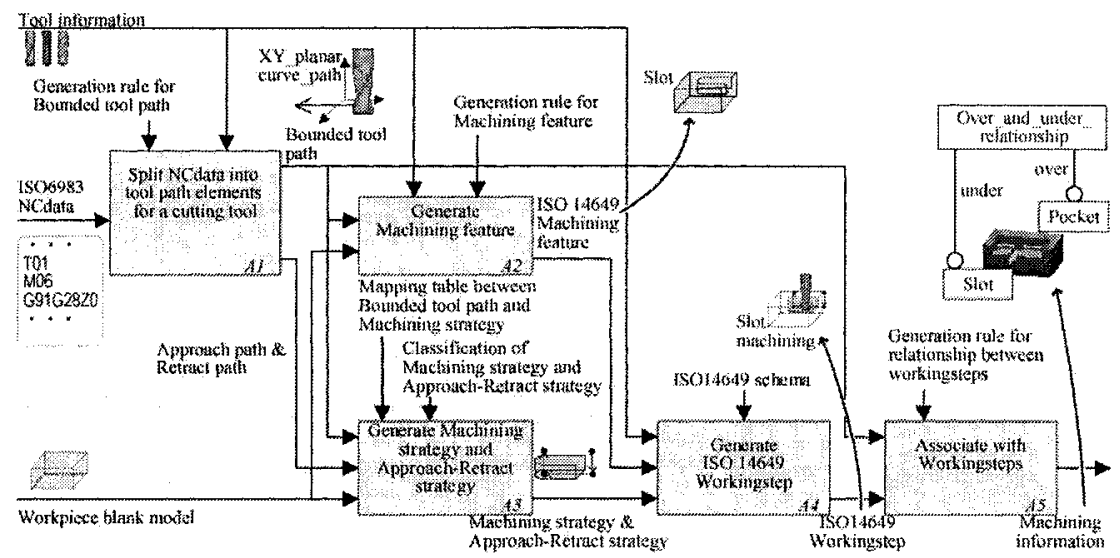

Figure 2. Procedure of analysis based on ISO 14649

\subsection{Generating machining feature from Bounded tool path data}

The activity of generating the machining feature from the bounded tool path, workpiece blank model, and tool information is shown in Figure 3 based on the form-shaping function ${ }^{5}$. In the first activity, the working tool is generated from the tool information and bounded tool path. The working tool is a functional model of the cutting edge generated from moving the macro tool that is generated by rotating the cutting edge around the spindle axis along the bounded tool path. The working tool is represented by the sweep profile which is the contact portion between the macro tool and the required shape and the sweep path which is the bounded tool path. The working edge is derived from the shape of the cutting tool. In the second activity, the removal volume is generated from the working tool. The removal volume is the subtracted volume of the working tool from the raw stock in the setup placement. To reflect the machining process information via the working tool, the removal volume inherits the information of the working tool and adds the boundary elements between the removal volume and the raw stock. In the third activity, the machining feature is generated from the removal volume. The machining feature is represented using the path and the profile in ISO $14649^{4}$. Therefore mapping between the 
machining feature and the removal volume which is represented as the pathprofile is possible.

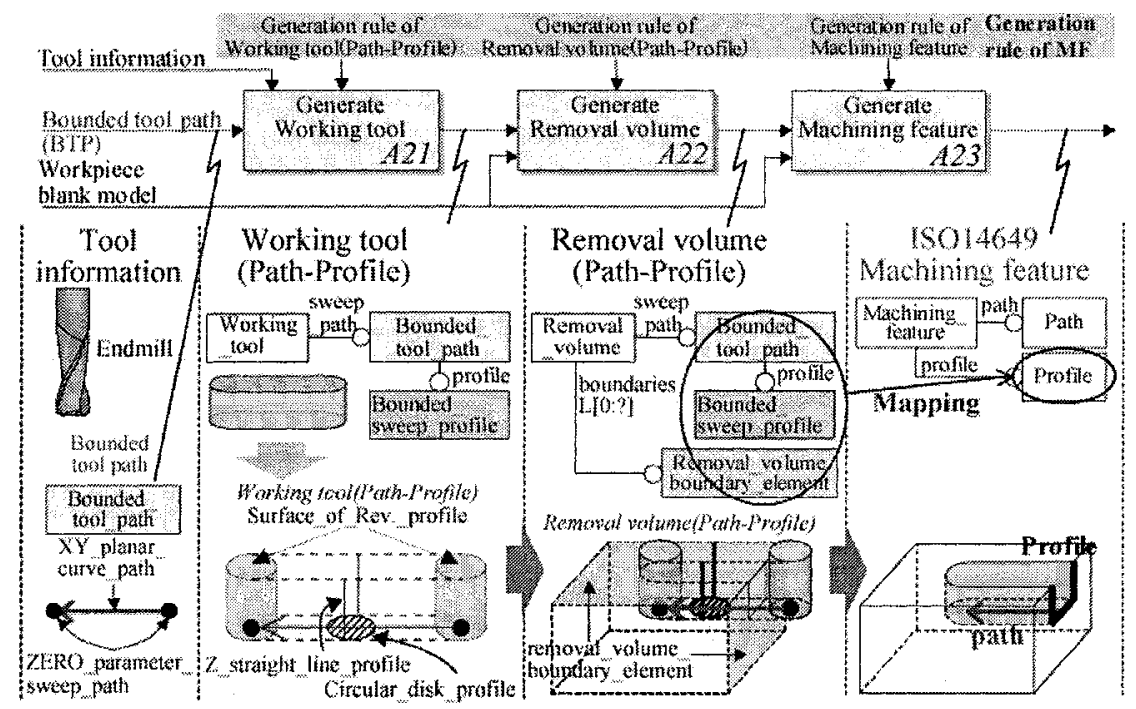

Figure 3. Generating machining feature from Bounded tool path

\subsection{Representation of the Relationship between Workingsteps}

In order to perform the process planning using the retained machining information, it is necessary to represent the relationship between workingsteps. However, in ISO 14649, there are few constructs which represent their relationships. Therefore, in this research, the new data model structure for describing the relationship between workingsteps is proposed as shown in Figure 4. In the proposed data structure, relationships consist of an operation relationship and a feature relationship.

The operation relationship describes precedence in machining processes. An example is the pocket which lays on the slot as shown in Figure 4 (c). In this case, the workingstep for pocket machining has priority over the one for slot machining. Such precedence is described by using Precedence relationship which is a subtype of Orientation relationship. In addition, Roughing_and_finishing which is a subtype of Precedence_relationship is used for the relationship between roughing and finishing.

The feature relationship describes the geometric positional relationship between machining features. It is defined based on ISO 10303 Part $108^{6}$. As 
shown in Figures 4 (a), (b) and (c), respectively, Parallel_relationship is used for the machining features which are positioned parallel, Coaxial relationship is used for the machining features which are coaxial, Over_and under_relationship is used for the machining features which exist one above the other.

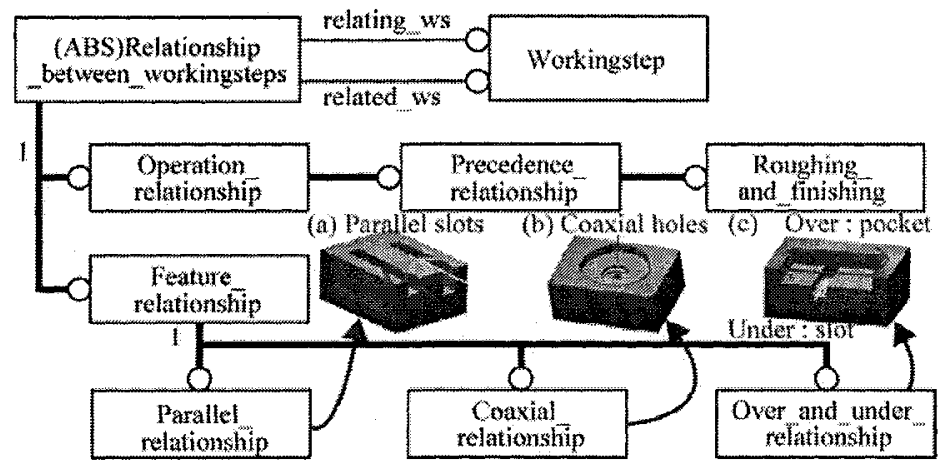

Figure 4. Data model structure for describing the relationship between workingsteps

\section{EXAMPLE}

In order to show the validity of the proposed system, we demonstrate how ISO $6983 \mathrm{NC}$ data generated by CAM software are applied to the implemented test system as shown in Figure 5.
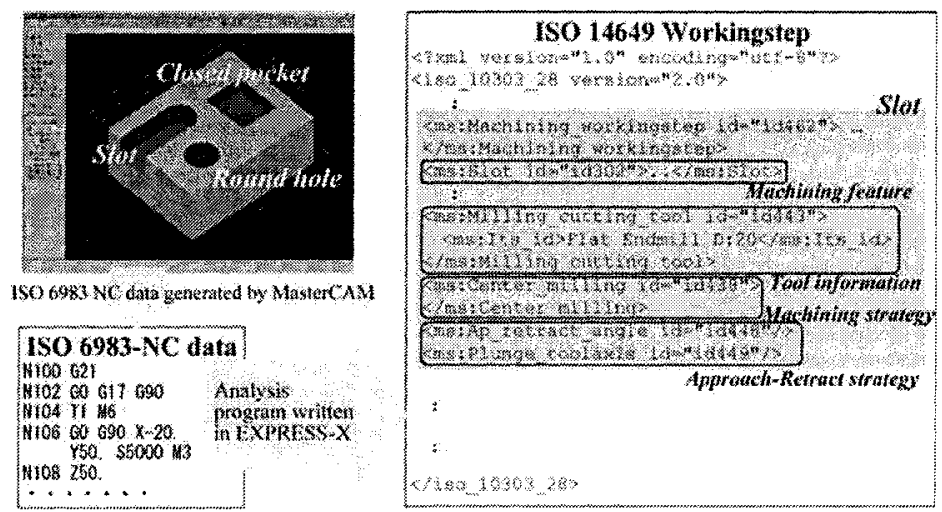

Figure 5. Data analysis example for slot, round hole, and closed pocket feature 
The test ISO $6983 \mathrm{NC}$ data for the slot, round hole, and closed pocket are generated by commercial CAM software (MasterCAM). As explained in previous sections, each activity in Figure 2 and Figure 3 is implemented by EXPRESS-X mapping language (EDMexpressX) ${ }^{7}$, and EPM EXPRESS Data manager to execute the mapping process is adopted. From this example, the Machining_workingstep for the Slot, the Machining_workingstep for the Closed pocket, and the Machining workingstep for the Round hole are obtained. These are the same as the contents of the ISO $6983 \mathrm{NC}$ data. Therefore, valid ISO $14649 \mathrm{NC}$ data are obtained.

\section{CONCLUSIONS}

In this research, technical information is extracted from the NC program, and is transformed into a next-generation CNC control language (ISO 14649) which contains the technical information. Conclusions of this research are as follows:

1. The method of extracting the machining feature and the process information from an $\mathrm{NC}$ program based on form-shaping function is proposed.

2. An analysis system for ISO $6983 \mathrm{NC}$ data based on ISO 14649 is implemented using EXPRESS-X.

3. ISO $6983 \mathrm{NC}$ data generated by CAM software are applied to the implemented test system, and valid ISO 14649 NC data are obtained.

\section{REFERENCES}

I. Yan X, Yamazaki K, and Liu J, Recognition of machining features and feature topologies from NC programs, Computer-Aided Design 32(10), 605-616 (2000)

2. K.Nieda, H.Aoyama. Database Construction System of Cutting Techniques and Cutting Conditions Based on Skilled Worker's Operation and NC Data. Proc. of the International Conference on Leading Edge Manufacturing in 21st Century, 67-72 (2005)

3. ISO 6983-1 Numeric control of machines-Program format and definition of address wordsPart 1: Data format for positioning, line motion and contouring control systems (1982)

4. ISO 14649-10: Industrial automation systems and integration- Physical device control-Data model for computerized numerical controllers-Part 10: General process data (2003)

5. Tanaka F., Takinami M., Kishinami T., Removal Volume Modeling for Process Planning Based on form-shaping function. Proc. of the International Conference on Leading Edge Manufacturing in 21 st Century, 747-752 (2003)

6. ISO10303-108: Industrial automation systems and integration-, Product data representation and exchange-, Part 108, Parameterization and Constraints for Explicit Geometric Product Models (2004)

7. EPM Technology, EDMassist Volume VI, EDMexpressX Language Reference (2002) 